\title{
AVALIAÇÃO DE PRODUTOS ALTERNATIVOS NO CONTROLE DE PRAGAS E NA QUALIDADE FISIOLÓGICA DE SEMENTES DE FEIJÃO MACASSAR ARMAZENADAS ${ }^{1}$
}

\author{
Hosaneide Farias Lima², Riselane de Lucena Alcântara Bruno³, Genildo Bandeira Bruno ${ }^{3}$ \\ e Ivanoska Salgado de Assis Bandeira ${ }^{4}$
}

\begin{abstract}
RESUMO
Avaliou-se a eficiência de produtos naturais e do produto químico fosfeto de alumínio, na qualidade fisiológica e sanitária das sementes de feijão macassar (Vigna unguiculata L. Walp.) variedade Cariri, no município de Solânea, PB, durante seis meses de armazenamento. Empregou-se o delineamento estatístico inteiramente casualizado, com arranjo fatorial $9 \times 7$, representado por nove tratamentos e sete períodos de armazenamento, com quatro repetições. A qualidade fisiológica das sementes foi avaliada no início e a cada 30 dias de armazenamento, através dos parâmetros: teor de umidade, índice de infestação, germinação e vigor (emergência em campo). Os resultados permitiram concluir que houve redução na qualidade fisiológica das sementes de feijão, ao longo do armazenamento; a casca de laranja-cravo e a pimenta- do-reino, moídas, foram os produtos que se revelaram mais eficazes no controle da infestação das sementes; apesar da redução na qualidade fisiológica das sementes armazenadas, a casca de laranja-cravo moída influenciou na melhoria da qualidade fisiológica das mesmas.
\end{abstract}

Palavras-chave: armazenamento, Vigna unguiculata L., controle de pragas

\section{EVALUATION OF ALTERNATIVE PRODUCTS FOR PLAGUE CONTROL AND PHYSIOLOGICAL QUALITY OF STORED COWPEA SEEDS}

\begin{abstract}
The efficiency of natural products and chemical aluminum phosphite, in the physiological and sanitary quality of stored cowpea seeds (Vigna unguiculata L. Walp.) variety Cariri, in the municipality of Solanea City, PB during six months of storage was evaluated. A completely randomized statistical design was used in a 9x7 factorial scheme represented by nine treatments and seven storage periods, with four replications. The physiological quality of the seeds was checked from the beginning up to 30 days of storage and the following parameters were evaluated: mean moisture content, infestation rate, germination and vigour (emergence in field). The results allowed to conclude that the cowpea seeds, during storage presented a reduced physiological quality. The most efficient alternative products for controlling infestation on seeds were crushed orange peel and ground black pepper. In spite of reduction in the physiological quality of stored seeds, the crashed orange peel was found to influence their quality.
\end{abstract}

Key words: storage, Vigna unguiculata L., plague control

\footnotetext{
${ }^{1}$ Parte da Dissertação de Mestrado do primeiro autor

${ }^{2}$ Eng. Agrônomo, M.Sc em Engenharia Agrícola. Rua Arlinda Gomes de Medeiros 97, Mirante, Campina Grande, PB

${ }^{3}$ Professores Doutores do Departamento de Fitotecnia, CCA - UFPb/ Areia, PB. e-mail: bruno@cca.ufpb.br

${ }^{4}$ Eng. Florestal, M.Sc em Engenharia Agrícola. Rua Lino Gomes Filho 255, Santo Antônio, Campina Grande, PB e-mail: bandeira@netwaybbs.com.br
} 


\section{INTRODUÇÃO}

O feijão macassar (Vigna unguiculata L. Walp.) é cultivado em todo o norte e nordeste do Brasil, constituindo-se importante fonte de renda e de subsistência para os pequenos agricultores; contudo, o nível de tecnologia empregado e as perdas de grãos que se intensificam não só em condições de campo, na época da colheita, mas principalmente durante as fases de secagem, beneficiamento e armazenamento do produto, têm determinado o baixo rendimento da cultura. Na fase de armazenamento, o ataque de insetos assume capital importância pelas perdas significativas dos produtos destinados à alimentação humana $\mathrm{e}$ animal (Braccini \& Picanço, 1995).

No armazenamento das sementes de feijão macassar, é fundamental ter em conta a infestação do lote, principalmente o gorgulho ou caruncho, Callosobruchus maculatus (Fab.) considerado a principal praga de armazenamento, danificando especialmente as sementes, em razão da baixa resistência que as mesmas oferecem contra essas pragas (Vieira et al., 1975).

$\mathrm{O}$ ataque de insetos em sementes armazenadas constitui um problema que se agrava cada vez mais devido, principalmente, ao desconhecimento dos produtores quanto à utilização e ao manuseio de substâncias químicas. Esses fatos conduzem à necessidade de se estabelecer medidas de controle de pragas a nível de fazenda, através de métodos alternativos, sem desencadear problemas provocados pelos inseticidas sintéticos químicos. Em um ecossistema onde os produtos químicos são aplicados, pode resultar na contaminação ambiental e, em conseqüência, provocar danos à saúde humana, poluir os recursos hídricos, provocar o surgimento de insetos resistentes e deixar resíduos tóxicos para o ser humano, além de onerar os custos de produção (Faroni et al., 1995).

Ante do exposto, o trabalho teve como objetivo avaliar a eficiência de produtos alternativos utilizados no tratamento das sementes de feijão macassar (Vigna unguiculata L. Walp.) variedade Cariri, no controle de pragas e na conservação da qualidade fisiológica das sementes armazenadas.

\section{MATERIAL E MÉTODOS}

O experimento referente ao armazenamento foi realizado no município de Solânea, PB, no período de maio a novembro de 1997.

Sementes de feijão macassar, variedade Cariri, foram tratadas com produtos naturais e com produto químico à base de fosfeto de alumínio (Tabela 1) acondicionadas em silos metálicos

Tabela 1. Produtos utilizados no tratamento de sementes de feijão

\begin{tabular}{lr}
\hline Produto & Quantidade \\
\hline $\mathrm{T}_{1}$ - Cinza de lenha & $300 \mathrm{~g}$ \\
$\mathrm{~T}_{2}$ - Casca de laranja cravo (pó) & $6 \mathrm{~g}$ \\
$\mathrm{~T}_{3}$ - Folhas de eucalipto (pó) & $6 \mathrm{~g}$ \\
$\mathrm{~T}_{4}$ - Folhas de fumo moídas (pó) & $6 \mathrm{~g}$ \\
$\mathrm{~T}_{5}$ - Produto químico à base de fosfeto de alumínio, & $15 \mathrm{~g}$ \\
$\mathrm{~T}_{6}$ - Óleo de soja & $10,5 \mathrm{~mL}$ \\
$\mathrm{~T}_{7}$ - Pimenta do reino (pó) & $6 \mathrm{~g}$ \\
$\mathrm{~T}_{8}$ - Vácuo (ausência de oxigênio) & \\
$\mathrm{T}_{9}$ - Testemunha (sem tratamento) & - \\
\hline
\end{tabular}

(21,1 cm de diâmetro e 19,5 cm de altura) e mantidas em armazém sob condições ambientais. Antes do armazenamento e a cada 30 dias, enviou-se um silo de cada tratamento, ao Laboratório de Análise de Sementes, da Universidade Federal da Paraíba do CCA - Areia, PB, para realização das análises, segundo as Regras para Análise de Sementes (Brasil, 1992).

\section{Teor de umidade}

$\mathrm{O}$ teor de umidade das sementes foi determinado pelo método da estufa a $105 \pm 3^{\circ} \mathrm{C}$ durante 24 horas, efetuando-se duas repetições de $50 \mathrm{~g}$ por tratamento.

\section{Índice de infestação}

$\mathrm{O}$ exame de infestação foi realizado utilizando-se quatro subamostras de 100 sementes por repetição/tratamento; as sementes foram observadas individualmente, para constatação de ovos, larvas, lagartas e insetos adultos, bem como orifícios de saída de insetos. O resultado foi expresso em percentagem de sementes infestadas.

\section{Teste padrão de germinação}

Foram utilizadas 200 sementes por tratamento (quatro repetições de 50 sementes) incubadas em rolo de papel Germitest, umedecido com água destilada colocando-se posteriormente no germinador, a $25^{\circ} \mathrm{C}$. A avaliação foi realizada aos cinco e oito dias de semeadura.

\section{Vigor - Emergência em campo}

Este teste foi conduzido em uma área experimental do Departamento de Fitotecnia do Centro de Ciências Agrárias da Universidade Federal da Paraíba, Areia-PB. Utilizaram-se 200 sementes (4 repetições de 50 sementes) distribuídas ao acaso, em sulcos distanciados de $0,20 \mathrm{~cm}$, com profundidade de semeadura de $0,20 \mathrm{~cm}$; as observações e contagem das plântulas normais foram realizadas a partir do quarto dia de instalação do teste, até permanecerem constantes, para calcular o Índice de Velocidade de Emergência em Campo (IVEC) de acordo com Vieira \& Carvalho (1994).

\section{Análise estatística}

Utilizou-se o delineamento inteiramente casualizado, em esquema fatorial $9 \times 7$ ( 9 tratamentos das sementes $\times 7$ períodos de armazenamento) com quatro repetições. Os dados expressos em porcentagem, para efeito da análise de variância, foram transformados em arc sen $\sqrt{\% 100}$, a fim de ocorrer a homogeneização das variações (Ferreira, 1996). A comparação entre os tratamentos realizou-se pelo teste de Tukey, a nível de $5 \%$ de probabilidade.

\section{RESULTADOS E DISCUSSÃO}

Os dados médios mensais de temperatura, precipitação pluviométrica e umidade relativa do local de armazenamento (Solânea, PB) das sementes de feijão, estão apresentados na Tabela 2 e os teores de umidade encontram-se na Figura 1. 
Tabela 2. Dados meteorológicos correspondentes aos meses de maio a novembro de 1997, no município de Solânea, PB.

\begin{tabular}{|c|c|c|c|c|c|}
\hline \multirow[t]{2}{*}{ Mês } & \multicolumn{3}{|c|}{ Temperatura $\left({ }^{\circ} \mathrm{C}\right)$} & \multirow{2}{*}{$\begin{array}{l}\text { Precipitação } \\
(\mathrm{mm})\end{array}$} & \multirow{2}{*}{$\begin{array}{c}\text { Umidade } \\
\text { Relativa \% }\end{array}$} \\
\hline & Máx & Min & Méd & & \\
\hline Maio & 26,8 & 20,4 & 26,3 & 4,4 & 80,1 \\
\hline Junho & 26,4 & 19,2 & 23,5 & 1,4 & 77,3 \\
\hline Julho & 25,9 & 18,4 & 23,1 & 2,7 & 79,3 \\
\hline Agosto & 26,5 & 18,7 & 21,8 & 1,5 & 74,9 \\
\hline Setembro & 29,4 & 19,4 & 22,4 & 0,42 & 64,3 \\
\hline Outubro & 30,7 & 20,2 & 24,6 & 0,029 & 62,2 \\
\hline Novembro & 31,5 & 20,5 & 25,7 & 0,06 & 61,3 \\
\hline
\end{tabular}

FONTE: Departamento de Ciências Atmosféricas da UFPB

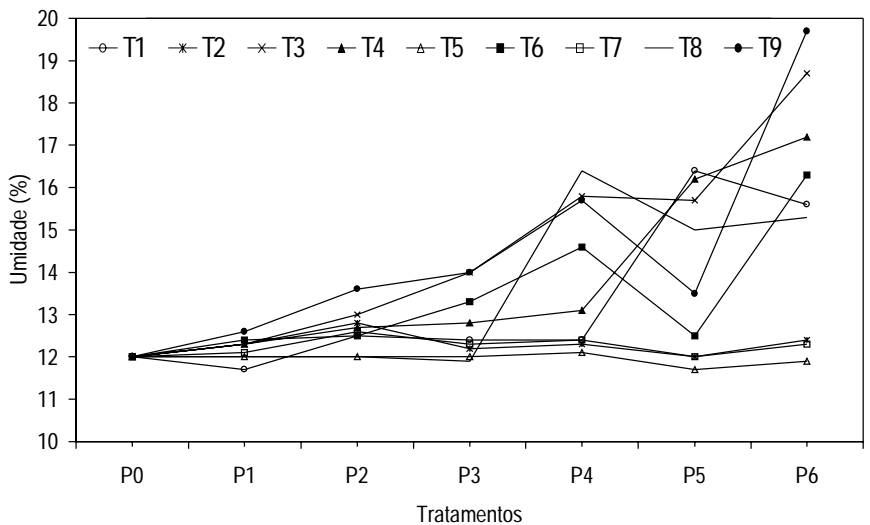

Figura 1. Médias mensais de teores de umidade das sementes de feijão, submetidas a tratamentos alternativos, durante seis meses de armazenamento, no município de Solânea, PB.

Durante o período de armazenamento (Tabela 2) a temperatura situou-se em uma amplitude de variação entre 18,4 e $31,5^{\circ} \mathrm{C}$, a umidade relativa reduziu de 80,1 a $61,3 \%$ e as precipitações entre 4,4 e 0,06 mm mensais. Observou-se que as condições climáticas iniciais não foram ótimas para o armazenamento e, ao se observar os teores de umidade das sementes nos diversos períodos de armazenamento (Figura 1) verificou-se, independentemente das condições climáticas predominantes, que sementes tratadas com produtos à base de casca de laranja-cravo $\left(\mathrm{T}_{2}\right)$, pimenta-doreino $\left(\mathrm{T}_{7}\right)$ e o produto químico fosfeto de alumínio $\left(\mathrm{T}_{5}\right)$ mantiveram teores de umidade estáveis ao longo do armazenamento enquanto aquelas submetidas ao tratamento de vácuo $\left(\mathrm{T}_{8}\right)$ e folhas de fumo $\left(\mathrm{T}_{4}\right)$ mantiveram a umidade constante até o terceiro e quarto períodos, respectivamente. Esses resultados estão de acordo com Germano (1997) ao afirmar que os produtos naturais alternativos aplicados às sementes de feijão macassar proporcionaram redução no teor de umidade das sementes acondicionadas em recipiente metálico; por outro lado, os demais tratamentos apresentaram teores de umidade elevados (acima de 13\%).

Com relação aos testes ensaiados, a análise de variância revelou efeitos altamente significativos para todos os parâmetros analisados, com exceção da interação no tocante à percentagem de emergência em campo, que não mostrou resposta significativa.

\section{Índice de infestação}

De acordo com a Tabela 3 verificou-se, ao longo do armazenamento, que as sementes apresentaram infestação mais elevada aos 150 dias $\left(\mathrm{P}_{5}\right)$. Dentre as substâncias aplicadas às sementes, a casca da laranja moída $\left(\mathrm{T}_{2}\right)$ foi mais eficiente no controle da infestação em relação aos demais tratamentos, confirmando o que já fora observado por Germano (1997) no tratamento de sementes de feijão Vigna.

$\mathrm{O}$ tratamento à base de pimenta-do-reino $\left(\mathrm{T}_{7}\right)$ também foi eficiente no combate à infestação por insetos, exibindo pequenos valores ao longo do armazenamento, o qual não diferiu do produto químico utilizado $\left(\mathrm{T}_{5}\right)$. Goldfarb (1997) trabalhando com sementes de milho armazenadas com extrato derivado de pimenta-do-reino, obteve $100 \%$ de controle de adultos de Sitophilus spp. Cabe ressaltar que as sementes de feijão utilizadas neste trabalho, tratadas com os produtos cascas de laranja $\left(\mathrm{T}_{2}\right) \mathrm{e}$ pimenta-do-reino $\left(\mathrm{T}_{7}\right)$, comparados ao fosfeto de alumínio $\left(\mathrm{T}_{5}\right)$, apresentaram menor percentagem de infestação e, consequentemente, manutenção do teor de umidade das sementes armazenadas.

As sementes tratadas com folhas de eucalipto $\left(\mathrm{T}_{3}\right)$ e folhas de fumo $\left(\mathrm{T}_{4}\right)$ sofreram crescente aumento no índice de infestação

Tabela 3. Índice de infestação das sementes de feijão submetidas a diferentes tratamentos, durante seis meses de armazenamento, no município de Solânea, PB.

\begin{tabular}{|c|c|c|c|c|c|c|c|c|c|c|}
\hline \multirow[t]{2}{*}{ Dias } & \multicolumn{9}{|c|}{ Tratamentos* } & \multirow[t]{2}{*}{ Média** } \\
\hline & $\mathrm{T}_{1}$ & $\mathrm{~T}_{2}$ & $\mathrm{~T}_{3}$ & $\mathrm{~T}_{4}$ & $\mathrm{~T}_{5}$ & $\mathrm{~T}_{6}$ & $\mathrm{~T}_{7}$ & $\mathrm{~T}_{8}$ & $\mathrm{~T}_{9}$ & \\
\hline 0 & $13,55 \mathrm{dA}$ & $13,55 \mathrm{abA}$ & $13,55 \mathrm{dA}$ & $13,55 \mathrm{cA}$ & $13,55 \mathrm{cA}$ & $13,55 \mathrm{cA}$ & $13,55 \mathrm{bcA}$ & $13,55 \mathrm{dA}$ & $13,55 \mathrm{dA}$ & $13,55 \mathrm{f}$ \\
\hline 60 & $20,80 \mathrm{dC}$ & $20,20 \mathrm{aC}$ & $57,36 \mathrm{cA}$ & $34,44 \mathrm{bB}$ & $21,38 \mathrm{bC}$ & $21,27 \mathrm{cC}$ & $20,01 \mathrm{bC}$ & $15,54 \mathrm{dC}$ & $52,44 \mathrm{cA}$ & $29,27 \mathrm{e}$ \\
\hline 90 & $29,07 \mathrm{cE}$ & $8,85 \mathrm{bF}$ & $69,90 \mathrm{bB}$ & 39,46 bD & $11,77 \mathrm{cF}$ & $82,60 \mathrm{aA}$ & $15,25 \mathrm{bcF}$ & $54,61 \mathrm{cC}$ & $74,15 \mathrm{bB}$ & $42,85 \mathrm{~d}$ \\
\hline 150 & $74,50 \mathrm{aC}$ & $13,60 \mathrm{abE}$ & $90,00 \mathrm{aA}$ & $90,00 \mathrm{aA}$ & $32,38 \mathrm{aD}$ & $83,90 \mathrm{aAB}$ & $18,78 \mathrm{bE}$ & 85,68 bAB & $77,85 \mathrm{bBC}$ & $62,96 \mathrm{a}$ \\
\hline 180 & $55,10 \mathrm{bB}$ & $8,85 \mathrm{bC}$ & 83,19 aA & $90,00 \mathrm{aA}$ & $13,55 \mathrm{cC}$ & $90,00 \mathrm{aA}$ & $14,90 \mathrm{bcC}$ & $90,00 \mathrm{aA}$ & $90,00 \mathrm{aA}$ & $59,51 \mathrm{~b}$ \\
\hline Média*** & $34,62 \mathrm{C}$ & $13,31 \mathrm{E}$ & $56,46 \mathrm{~A}$ & $52,91 \mathrm{~B}$ & $17,83 \mathrm{D}$ & $50,06 \mathrm{~B}$ & $17,83 \mathrm{D}$ & $50,29 \mathrm{~B}$ & $58,29 \mathrm{~A}$ & \\
\hline
\end{tabular}

(*) Tratamentos: $T_{1}$ - cinza de lenha, $T_{2}$ - casca da laranja, $T_{3}$ - folha de eucalipto, $T_{4}$ - folha de fumo, $T_{5}$ - fosfeto de alumínio $T_{6}$ - óleo de soja, $T_{7}$ - pimenta-do-reino, $T_{8}$ - vácuo, $T_{9}$ - testemunha DMS

$(* *)$ Médias seguidas pela mesma letra minúscula na coluna $\left(\mathrm{DMS}_{\text {columa }}=7,76\right)$ e maiúsculas na linha $\left(\mathrm{DMS}_{\text {linha }}=8,17\right)$ não diferem estatisticamente pelo teste de Tukey a $5 \%$ 
aos 60 dias de armazenamento, apresentando níveis mais elevados a partir dos $150\left(\mathrm{P}_{5}\right)$ e 120 dias $\left(\mathrm{P}_{4}\right)$, respectivamente. No material tratado com cinzas de lenha $\left(\mathrm{T}_{1}\right)$, óleo de soja $\left(\mathrm{T}_{6}\right)$ e vácuo $\left(\mathrm{T}_{8}\right)$ o aumento no índice de infestação ocorreu a partir dos 90 dias de armazenamento $\left(\mathrm{P}_{3}\right)$; por outro lado, o tratamento menos eficiente no controle da infestação média de insetos foi aquele à base de folha de eucalipto $\left(\mathrm{T}_{3}\right)$ estatisticamente igual às sementes não tratadas $\left(\mathrm{T}_{9}\right)$. Esta ocorrência contradiz as observações feitas por Rodrigues \& Benedetti (1997) e Germano (1997) quando armazenaram sementes de feijão macassar tratadas com folhas de eucalipto e observaram o controle das pragas.

\section{Germinação}

Analisando-se as interações tratamentos x períodos (Tabela 4) constatou-se, no decorrer de 120 dias de armazenamento $\left(\mathrm{P}_{4}\right)$ que a porcentagem de germinação $(63 \%)$ foi superior em todos os tratamentos, inclusive no período inicial (54\%). Embora tendo ocorrido este aumento na germinação verificou-se, de modo geral, que a viabilidade dessas sementes se encontra abaixo do padrão mínimo estabelecido pela legislação estadual (CESM, 1989) a qual recomenda, para o feijão macassar, uma germinação de $70 \%$. Dos tratamentos utilizados, os melhores resultados foram alcançados com sementes tratadas com cascas de laranja $\left(\mathrm{T}_{2}\right)$ seguido dos tratamentos à base de pimenta-do-reino $\left(\mathrm{T}_{7}\right)$, vácuo $\left(\mathrm{T}_{8}\right)$, folhas de fumo $\left(\mathrm{T}_{4}\right)$ e o produto químico $\left(\mathrm{T}_{5}\right)$. A menor eficiência dos tratamentos foi verificada a partir dos 150 dias de armazenamento $\left(\mathrm{P}_{5}\right)$ perdendo totalmente sua capacidade de germinação. Segundo Popinigis (1985) a perda de vigor decorre de um processo natural de deterioração, e a germinação é a última medida de qualidade a declinar em sementes.

Ao que parece, os tratamentos naturais aplicados às sementes não influenciaram de maneira significativa na manutenção da germinação das mesmas, quando comparadas com as sementes não tratadas, provavelmente em função da sua baixa qualidade fisiológica. Considerando-se os valores médios, verifica-se ainda que, apesar da baixa qualidade dessas sementes, o tratamento casca de laranja moída $\left(\mathrm{T}_{2}\right)$ foi o que diferiu estatisticamente da testemunha $\left(\mathrm{T}_{9}\right)$. Cavalcanti Mata (1987) relata que sementes de feijão macassar tratadas com casca de laranja seca e moída, não apresentaram alterações na qualidade durante longos períodos de armazenamento e que a ação do ácido cítrico pode não estar restrito apenas à manutenção da coloração das sementes, mas estendido também à dureza do tegumento. Germano (1997) e Almeida et al. (1994) trataram sementes de feijão Vigna com extratos brutos da casca de laranja e obtiveram bons resultados na manutenção da germinação e do vigor dessas sementes.

\section{Vigor - Emergência em campo}

Os dados referentes à emergência de plântulas em campo

Tabela 4. Valores médios de germinação das sementes de feijão submetidas a diferentes tratamentos, durante seis meses de armazenamento, no município de Solânea, PB

\begin{tabular}{cccccccccccc}
\hline Dias & \multicolumn{9}{c}{ Tratamentos* } & \multicolumn{1}{c}{ Média** } \\
\cline { 2 - 8 } & \multicolumn{1}{c}{$\mathrm{T}_{1}$} & \multicolumn{1}{c}{$\mathrm{T}_{2}$} & $\mathrm{~T}_{3}$ & $\mathrm{~T}_{4}$ & $\mathrm{~T}_{5}$ & $\mathrm{~T}_{6}$ & $\mathrm{~T}_{7}$ & $\mathrm{~T}_{8}$ & $\mathrm{~T}_{9}$ \\
\hline 0 & $47,27 \mathrm{abA}$ & $47,27 \mathrm{abA}$ & $47,27 \mathrm{abA}$ & $47,27 \mathrm{abA}$ & $47,27 \mathrm{bA}$ & $47,27 \mathrm{aA}$ & $47,27 \mathrm{abA}$ & $47,27 \mathrm{abA}$ & $47,27 \mathrm{abA}$ & $47,27 \mathrm{~b}$ \\
30 & $45,58 \mathrm{bcA}$ & $47,35 \mathrm{abA}$ & $41,18 \mathrm{bcA}$ & $48,79 \mathrm{abA}$ & $41,77 \mathrm{bcA}$ & $31,23 \mathrm{bB}$ & $42,99 \mathrm{bcA}$ & $46,16 \mathrm{abA}$ & $40,88 \mathrm{bcA}$ & $42,88 \mathrm{c}$ \\
60 & $47,30 \mathrm{abA}$ & $51,27 \mathrm{aA}$ & $47,02 \mathrm{abA}$ & $44,71 \mathrm{abA}$ & $44,14 \mathrm{bA}$ & $43,57 \mathrm{aA}$ & $45,00 \mathrm{bcA}$ & $47,59 \mathrm{abA}$ & $43,25 \mathrm{abcA}$ & $45,98 \mathrm{~b}$ \\
90 & $38,34 \mathrm{cAB}$ & $42,70 \mathrm{bAB}$ & $38,34 \mathrm{cAB}$ & $42,99 \mathrm{bAB}$ & $35,66 \mathrm{cB}$ & $35,63 \mathrm{bB}$ & $38,64 \mathrm{cAB}$ & $44,13 \mathrm{bA}$ & $38,63 \mathrm{cAB}$ & $39,45 \mathrm{~d}$ \\
120 & $53,23 \mathrm{aA}$ & $54,67 \mathrm{aA}$ & $50,02 \mathrm{aA}$ & $51,38 \mathrm{aA}$ & $55,27 \mathrm{aA}$ & $49,91 \mathrm{aA}$ & $54,11 \mathrm{aA}$ & $52,65 \mathrm{aA}$ & $50,27 \mathrm{aA}$ & $52,39 \mathrm{a}$ \\
150 & $0,57 \mathrm{dD}$ & $21,30 \mathrm{cAB}$ & $13,52 \mathrm{dBC}$ & $13,53 \mathrm{cBC}$ & $19,68 \mathrm{dABC}$ & $16,65 \mathrm{cABC}$ & $23,42 \mathrm{dA}$ & $12,20 \mathrm{cC}$ & $12,30 \mathrm{dC}$ & $14,69 \mathrm{e}$ \\
180 & $0,57 \mathrm{dA}$ & $0,57 \mathrm{Da}$ & $0,57 \mathrm{dA}$ & $0,57 \mathrm{dA}$ & $0,57 \mathrm{eA}$ & $0,57 \mathrm{dA}$ & $0,57 \mathrm{eA}$ & $0,57 \mathrm{dA}$ & $0,57 \mathrm{eA}$ & $0,57 \mathrm{f}$ \\
\hline Média** & $33,26 \mathrm{BC}$ & $37,87 \mathrm{~A}$ & $33,99 \mathrm{BC}$ & $35,60 \mathrm{AB}$ & $34,90 \mathrm{ABC}$ & $31,98 \mathrm{C}$ & $36,00 \mathrm{AB}$ & $35,79 \mathrm{AB}$ & $33,31 \mathrm{BC}$ & \\
\hline
\end{tabular}

(*) Tratamentos: $T_{1}$ - cinza de lenha, $T_{2}$ - casca da laranja, $T_{3}$ - folha de eucalipto, $T_{4}$ - folha de fumo, $T_{5}$ - fosfeto de alumínio $T_{6}$ - óleo de soja, $T_{7}-$ pimenta-do-reino, $T_{8}$ - vácuo, $T_{9}-$ testemunha; DMS tratamento $=3,03$ DMS ${ }_{\text {perído }}=2,54$

(**) Médias seguidas pela mesma letra minúscula na coluna $\left(\mathrm{DMS}_{\text {columa }}=7,62\right)$ e maiúsculas na linha $\left(\mathrm{DMS}_{\text {linha }}=8,03\right)$ não diferem estatisticamente pelo teste de Tukey a $5 \%$

Tabela 5. Valores médios do vigor (emergência em campo) das sementes de feijão submetidas a diferentes tratamentos, durante seis meses de armazenamento, no município de Solânea, PB

\begin{tabular}{|c|c|c|c|c|c|c|c|c|c|c|}
\hline \multirow[t]{2}{*}{ Dias } & \multicolumn{9}{|c|}{ Tratamentos* } & \multirow[t]{2}{*}{ Média** } \\
\hline & $\mathrm{T}_{1}$ & $\mathrm{~T}_{2}$ & $\mathrm{~T}_{3}$ & $\mathrm{~T}_{4}$ & $\mathrm{~T}_{4}$ & $\mathrm{~T}_{6}$ & $\mathrm{~T}_{7}$ & $\mathrm{~T}_{8}$ & $\mathrm{~T}_{9}$ & \\
\hline 0 & 2,39 & 2,39 & 2,39 & 2,39 & 2,39 & 2,39 & 2,39 & 2,39 & 2,39 & $2,39 \mathrm{c}$ \\
\hline 60 & 3,30 & 2,85 & 3,01 & 3,08 & 2,63 & 1,97 & 2,95 & 3,36 & 3,74 & $2,99 \mathrm{bc}$ \\
\hline 90 & 3,27 & 4,53 & 2,96 & 2,96 & 3,69 & 2,66 & 3,60 & 3,37 & 2,20 & $3,25 \mathrm{~b}$ \\
\hline 150 & 2,40 & 4,83 & 1,77 & 1,77 & 4,27 & 3,39 & 4,51 & 2,71 & 2,82 & $3,16 \mathrm{bc}$ \\
\hline 180 & 0,57 & 0,57 & 0,57 & 0,57 & 0,57 & 0,57 & 0,57 & 0,57 & 0,57 & $0,57 \mathrm{~d}$ \\
\hline Média** & $3,18 \mathrm{AB}$ & $3,58 \mathrm{~A}$ & $2,67 \mathrm{AB}$ & $2,91 \mathrm{AB}$ & $3,37 \mathrm{AB}$ & 2,64 B & $3,32 \mathrm{AB}$ & $2,85 \mathrm{AB}$ & $2,82 \mathrm{AB}$ & \\
\hline
\end{tabular}

(*) Tratamentos: $T_{1}$ - cinza de lenha, $T_{2}$ - casca da laranja, $T_{3}$ - folha de eucalipto, $T_{4}$ - folha de fumo, $T_{5}$ - fosfeto de alumínio $T_{6}$ - óleo de soja, $T_{7}-$ pimenta-do-reino, $T_{8}$ - vácuo, $T_{9}$ - testemunha $(* *)$ Médias seguidas pela mesma letra minúscula na coluna $\left(\mathrm{DMS}_{\text {coluna }}=0,78\right)$ e maiúsculas na linha $\left(\mathrm{DMS}_{\text {linha }}=0,94\right)$ não diferem estatisticamente pelo teste de Tukey a $5 \%$ 
(Tabela 5) mostram acréscimo do vigor aos 30 dias de armazenamento $\left(\mathrm{P}_{1}\right)$ diferindo estatisticamente dos demais períodos. Em seguida, decréscimo em função do tempo de armazenamento, evidenciando-se estabilização da emergência dessas plântulas dos 60 aos 150 dias de armazenamento $\left(\mathrm{P}_{2}, \mathrm{P}_{3}\right.$, $\mathrm{P}_{4}$ e $\mathrm{P}_{5}$ ) resultando em um decréscimo bastante acentuado aos 180 dias $\left(\mathrm{P}_{6}\right)$. Esses resultados estão de acordo com Figueirêdo et al. (1998) quando estudaram o armazenamento de sementes de arroz (Oryza sativa L.) durante seis meses, afirmando que o valor médio do vigor tende a decrescer significativamente ao longo do tempo.

Observou-se, no entanto, pela Tabela 5, que as sementes tratadas com casca de laranja moída $\left(\mathrm{T}_{2}\right)$ apresentaram o maior índice médio de emergência em campo, diferindo apenas das sementes tratadas com óleo de soja $\left(\mathrm{T}_{6}\right)$ onde se constatou o pior valor médio de vigor. Pelos resultados obtidos percebe-se, de modo geral, que não houve resposta dos tratamentos alternativos quanto ao vigor dessas sementes devido, provavelmente, à baixa qualidade fisiológica das mesmas.

\section{CONCLUSÕES}

De acordo com os resultados alcançados, pode-se concluir que:

1. Houve redução na qualidade fisiológica das sementes de feijão, ao longo do armazenamento.

2. A casca de laranja cravo e a pimenta-do-reino moídas, foram os produtos que se revelaram mais eficazes no controle da infestação das sementes.

3. Apesar da redução na qualidade fisiológica das sementes armazenadas, a casca de laranja cravo moída influenciou na melhoria da qualidade fisiológica das mesmas.

\section{REFERÊNCIAS BIBLIOGRÁFICAS}

ALMEIDA, F.A.C.; CAVALCANTI MATA, M.E.R.M. Avaliação dos componentes químicos do feijão macassar armazenado com extratos de casca de laranja e de limão. Campina Grande, UFPB/NTA, 1994. 18p. UFPB/NTA, Boletim Técnico, 14

BRACCINI, A.L.; PICANÇO, M. Manejo integrado de pragas do feijoeiro no armazenamento. Revista Brasileira de Armazenamento. Viçosa, v. 20, n.1/2, p.37-43, 1995.

BRASIL. Ministério da Agricultura. Regras para análise de sementes. Brasília: AGIPLAN, 1992. 188p.

CAVALCANTI MATA, M.E.R.M. Efeitos da secagem em altas temperaturas por curtos períodos de tempo, em camada estacionária, sobre a armazenabilidade de sementes de feijão (Phaseolus vulgaris L.) variedade "carioca": Avaliação experimental, modelagem e simulação. Campinas: Universidade Estadual de Campinas, 1997. 344p. Tese Doutorado.

CESM - Delegacia Federal de Agricultura na Paraíba - SEAPRO (DFA/PB). $3^{\text {a }}$ ed. João Pessoa, PB, 1989, 85p.

FARONI, L.R.A.; MOLIN, L.; ANDRADE, E.T.; CARDOSO, E.G. Utilização de produtos naturais no controle de Acanthoscelides obtectus em feijão armazenado. Revista Brasileira de Armazenamento. Viçosa, v.20, n.1/2, p.4448, 1995.

FERREIRA, P.V. Estatística experimental aplicada à agronomia. Alagoas: EDUFAL, 1996. 606p.

FIGUEIRÊDO, R.M.F.; CAVALCANTI MATA, M.E.R.M.; QUEIROGA, V.P. Germinação e vigor de sementes de arroz armazenadas em diferentes tipos de embalagem em três microrregiões do Estado da Paraíba. Revista Brasileira de Engenharia Agrícola e Ambiental. Campina Grande, v.2, n.1, p.84-88, 1998.

GERMANO, M.L.A.R. Emprego de produtos naturais no tratamento de sementes de feijão macassar (Vigna unguiculata (L.) Walp.) acondicionadas em três embalagens e em microrregiões do Estado da Paraíba. Areia: UFPB, 1997. 77p. Dissertação Mestrado.

GOLDFARB, A.C. Controle do inseto Sitophilus ssp com extratos naturais de origem vegetal e seus efeitos na qualidade fisiológica em sementes de milho. Campina Grande: UFPB, 1997. 77p. Dissertação Mestrado.

POPINIGIS, F. Fisiologia da semente. Brasília: AGIPLAN, 1985. 289p.

RODRIGUES, E.; BENEDETTI, B.C. Comparação entre folhas de eucalipto e inseticida para proteção do feijão (Phaseolus vulgaris L.) armazenado. In: CONGRESSO BRASILEIRO DE ENGENHARIA AGRÍCOLA, 26., 1997. Campina Grande. Resumos... Campina Grande: UFPB/SBEA, 1997. p. 10 .

VIEIRA, R.D.; CARVALHO, N.M. Teste de vigor em sementes. Viçosa: FUMEP, 1994. 164p.

VIEIRA, F.V.; BASTOS, J.A.M.; PEREIRA, L. Influência do Chalcodermus bimaculatus Fiedler, 1936 (Col., Curc) sobre o poder germinativo do feijão de corda, Vigna sinensis (L.) Savi. Fitossanidade. Fortaleza, v.1, n.2, p.47-8, 1975. 